\title{
A new fixed point result via property $P$ with an application
}

\author{
Zead Mustafa $^{\mathrm{a}, \mathrm{b}}$, M. M. M. Jaradat ${ }^{\mathrm{a}}$, Erdal Karapınar ${ }^{\mathrm{c}, \mathrm{d}, *}$ \\ ${ }^{a}$ Department of Mathematics, Statistics and Physics, Qatar University, Doha, Qatar. \\ ${ }^{b}$ Department of Mathematics, The Hashemite University, P. O. 330127, Zarqa 13115, Jordan. \\ ${ }^{c}$ Nonlinear Analysis and Applied Mathematics (NAAM) Research Group, KAU, 21589, Jeddah, Saudi Arabia. \\ ${ }^{d}$ Department of Mathematics, Atilim University 06836, Incek, Ankara, Turkey. \\ Communicated by M. Eslamian
}

\begin{abstract}
The purpose of this paper is to introduce a new contractive condition. We prove the existence and uniqueness of a fixed point of self-mapping under this new contractive condition. Moreover, we observe analog of these results for the mappings that satisfy the property P. An application on integral equations is presented to illustrate the main result. Our results extend and generalize well-known results in the literature. (C)2017 All rights reserved.
\end{abstract}

Keywords: Contractive mapping, fixed point, partial metric space, property $\mathrm{P}$, integral equations. 2010 MSC: 46T99, 47H10, 54H25.

\section{Introduction and mathematical preliminaries}

The notion of non-zero self-distance was initiated by Matthews [38] to solve the existing problems in the research area of the denotational semantics of dataflow network. By the help of that concept, the author extended the notion of metric, by introducing the notion of partial metric, to use the analog of well-known the Banach contraction mapping principle for his purpose, in particular, for program verification/termination.

Partial metric spaces become more useful research field after the contribution of Romaguera [44] to this theory, namely, the notions of 0 -completeness. Recently, a number of authors have focused on the fixed point results in partial metric spaces, see e.g. [1-18, 20-36, 41-45] and references therein.

The aim of this paper is to prove some fixed point results for self-mappings in partial metric space satisfying a new contractive condition, also we show that the mapping satisfies the property P. We give also an example to illustrate the main result. Moreover, we propose an application of integral equations that supports the presented results.

First, we recollect some basics notions and facts. Throughout the paper, let $\mathbb{N}, \mathbb{Q}, \mathbb{R}$ denote the natural, rational and real numbers, respectively. First, we recall the notion of a partial metric:

\footnotetext{
${ }^{*}$ Corresponding author

Email addresses: zead@qu .edu.qa (Zead Mustafa), mmjst4@qu.edu.qa (M. M. M. Jaradat), erdalkarapinar@yahoo.com (Erdal Karapınar)

doi:10.22436/jnsa.010.04.61
}

Received 2017-01-27 
Definition 1.1 ([38]). A partial metric on a nonempty set $X$ is a function $p: X \times X \rightarrow[0, \infty)$ such that

(P1) $p(x, x)=p(x, y)=p(y, y)$ if and only if $x=y$;

(P2) $p(x, x) \leqslant p(x, y)$;

(P3) $p(x, y)=p(y, x)$;

(P4) $p(x, y) \leqslant p(x, z)+p(z, y)-p(z, z)$,

hold for all $x, y, z \in X$. Here, we denote partial metric space by a pair $(X, p)$.

It is straightforward to see that the function $d_{p}: X \times X \rightarrow[0, \infty)$ given by

$$
d_{p}(x, y)=2 p(x, y)-p(x, x)-p(y, y),
$$

forms a standard (usual) metric on $X$.

Definition $1.2([38,42])$. Let $(X, p)$ be a partial metric space. Then

(1) A sequence $\left\{x_{n}\right\}$ in $(X, p)$ converges to a point $x \in X$ if and only if $p(x, x)=\lim _{n \rightarrow \infty} p\left(x_{n}, x\right)$.

(2) A sequence $\left\{x_{n}\right\}$ in $(X, p)$ is called a Cauchy sequence if $\lim _{n, m \rightarrow \infty} p\left(x_{n}, x_{m}\right)$ exists and finite.

(3) $(X, p)$ is said to be complete if every Cauchy sequence $\left\{x_{n}\right\}$ in $X$ converges with respect to its topology $\tau_{p}$ to a point $x \in X$ such that $p(x, x)=\lim _{n, m \rightarrow \infty} p\left(x_{n}, x_{m}\right)$.

(4) A sequence $\left\{x_{n}\right\}$ in $(X, p)$ is called a 0 -Cauchy sequence if $\lim _{n, m \rightarrow \infty} p\left(x_{n}, x_{m}\right)=0$. The space $(X, p)$ is said to be 0 -complete if every 0 -Cauchy sequence in $X$ converges with respect to $\tau_{p}$ to a point $x \in X$ such that $p(x, x)=0$.

Lemma $1.3([38,42])$. Let $(X, p)$ be a partial metric space and $\left\{x_{n}\right\}$ be any sequence in $X$. Then

(i) $\left\{x_{n}\right\}$ is a Cauchy sequence in $(X, p)$ if and only if it is a Cauchy sequence in the metric space $\left(X, d_{p}\right)$.

(ii) The space $(X, p)$ is complete if and only if the metric space $\left(X, \mathrm{~d}_{\mathrm{p}}\right)$ is complete.

(iii) Every 0-Cauchy sequence in $(X, p)$ is Cauchy in $\left(X, d_{p}\right)$.

(iv) If $(\mathrm{X}, \mathrm{p})$ is complete, then it is 0-complete.

The converse assertions of (iii) and (iv) do not hold as the following easy example shows.

Example 1.4 ([44]). The space $X=\mathbb{Q} \cap[0, \infty)$ with the partial metric $p(x, y)=\max \{x, y\}$ is 0 -complete but it is not complete (since $d_{\mathfrak{p}}(x, y)=|x-y|$ and $\left(X, d_{\mathfrak{p}}\right)$ is not complete). Moreover, the sequence $\left\{x_{n}\right\}$ with $x_{n}=1$ for each $n \in \mathbb{N}$ is a Cauchy sequence in $(X, p)$, but it is not a 0 -Cauchy sequence.

Notice also that every closed subset of a 0-complete partial metric space is 0-complete.

Lemma $1.5([1,42])$. Assume $\left\{x_{n}\right\} \rightarrow z$ as $\mathrm{n} \rightarrow \infty$ in a partial metric space $(X, p)$ such that $\mathrm{p}(z, z)=0$. Then $\lim _{n \rightarrow \infty} p\left(x_{n}, y\right)=p(z, y)$ for all $y \in X$.

Lemma $1.6([1,23])$. Suppose that $(X, p)$ is a partial metric space. Then

(1) If $p(x, y)=0$, then $x=y$.

(2) If $x \neq y$, then $p(x, y)>0$.

Definition $1.7([37])$. A function $\psi:[0, \infty) \rightarrow[0, \infty)$ is called an altering distance function if it satisfies the following conditions:

1. $\psi$ is continuous and nondecreasing;

2. $\psi(t)=0$ if and only if $t=0$.

We denote $\Psi$ the set of all altering distance functions. 
Definition 1.8. The function $\varphi:[0, \infty) \rightarrow[0, \infty)$ is called a strong-altering distance function, if the following conditions hold.

1. $\varphi$ is continuous;

2. $\varphi(t) \neq 0$ when $t \neq 0$.

We denote by $\Phi$ the set of all strong-altering distance functions.

Lemma 1.9 ([40]). Let $(X, p)$ be a partial metric space and let $\left\{x_{n}\right\}$ be a sequence in $X$ such that $\lim _{n \rightarrow \infty} p\left(x_{n}, x_{n+1}\right)=$ 0 . If $\left\{x_{2 n}\right\}$ is not a Cauchy sequence in $(X, p)$, then there exist $\varepsilon>0$ and two sequences $\{m(k)\}$ and $\{n(k)\}$ of positive integers such that $\mathrm{n}(\mathrm{k})>\mathrm{m}(\mathrm{k})>\mathrm{k}$ and the following four sequences tend to $\varepsilon>0$, when $\mathrm{k} \rightarrow \infty$.

$$
p\left(x_{2 m(k)}, x_{2 n(k)+1}\right), p\left(x_{2 m(k)}, x_{2 n(k)}\right), p\left(x_{2 m(k)-1}, x_{2 n(k)+1}\right), p\left(x_{2 m(k)-1}, x_{2 n(k)}\right) .
$$

The following lemmas will play crucial roles in the sequel.

Lemma 1.10 ([39]). Let $(X, p)$ be a partial metric space and $\left\{x_{n}\right\}$ be a sequence such that $\lim _{n \rightarrow \infty} p\left(x_{n}, x_{n+1}\right)=0$, then the sequence $\left\{x_{n}\right\}$ is a Cauchy sequence if and only if $\left\{x_{2 n}\right\}$ is a Cauchy subsequence.

Lemma 1.11 ([39]). Let $(X, p)$ be a complete partial metric space and $\left\{x_{n}\right\}$ be a Cauchy sequence such that $\lim _{n \rightarrow \infty} p\left(x_{n}, x_{n}\right)=0$, then the sequence $\left\{x_{n}\right\}$ is a 0-Cauchy sequence. Further, if $\left\{x_{n}\right\}$ converges to $x$, then $\lim _{n \rightarrow \infty} p\left(x_{n}, x\right)=0$.

Lemma 1.12 ([39]). Let $(X, p)$ be a complete partial metric space and $\left\{x_{n}\right\}$ be a Cauchy sequence such that $\lim _{n \rightarrow \infty} p\left(x_{n}, x_{n+1}\right)=0$. Then the sequence $\left\{x_{n}\right\}$ is a 0 -Cauchy sequence.

We should underline the fact that the limit of a sequence in partial metric space is not necessarily unique. For instance, the limit of the sequence $\left\{\frac{1}{n^{3}+1}\right\}_{n \in \mathbb{N}}$ in the partial metric space, $(X, p)$ defined by

$$
p(x, y)=\max \{x, y\} \text { for all } x, y \in X=[0, \infty),
$$

is not unique. More precisely, we have

$$
p(1,1)=\lim _{n \rightarrow \infty} p\left(1, \frac{1}{n^{3}+1}\right) \quad \text { and } \quad p(2,2)=\lim _{n \rightarrow \infty} p\left(2, \frac{1}{n^{3}+1}\right) .
$$

On the other hand, under the certain restriction, the uniqueness of a limit of a given sequence can be guaranteed. The following lemma is one of the example of how the uniqueness criteria can be provided.

Lemma 1.13 (See e.g. [34]). Let $(X, p)$ be a partial metric space and let $\left\{x_{n}\right\}_{n \in \mathbb{N}}$ be a sequence in $X$ such that $x_{n} \rightarrow x$ and $x_{n} \rightarrow y$. If

$$
\lim _{n \rightarrow \infty} p\left(x_{n}, x_{n}\right)=p(x, x)=p(y, y),
$$

then $x=y$.

\section{Main results}

We start this section with the following crucial lemma.

Lemma 2.1. Let $(X, p)$ be a partial metric space and let $\left\{x_{n}\right\}$ be a sequence in $X$ such that $\lim _{n \rightarrow \infty} p\left(x_{n}, x_{n+1}\right)=0$. If $\left\{x_{2 n}\right\}$ is not a Cauchy sequence in $(X, p)$, then there exist an $\varepsilon>0$ and two sequences $\{m(k)\}$ and $\{n(k)\}$ of positive integers such that $\mathrm{n}(\mathrm{k})>\mathrm{m}(\mathrm{k})>\mathrm{k}$ and

$$
\lim _{k \rightarrow \infty} p\left(x_{2 m(k)}, x_{2 n(k)-1}\right)=\varepsilon
$$


Proof. By Lemma 1.9 there exist $\varepsilon>0$ and two sequences $\{m(k)\}$ and $\{n(k)\}$ of positive integers such that $n(k)>m(k)>k$ and

$$
\lim _{k \rightarrow \infty} p\left(x_{2 n(k)}, x_{2 m(k)}\right)=\varepsilon
$$

From (P4), we have

$$
p\left(x_{2 m(k)}, x_{2 n(k)-1}\right) \leqslant p\left(x_{2 m(k)}, x_{2 n(k)}\right)+p\left(x_{2 n(k)}, x_{2 n(k)-1}\right)-p\left(x_{2 n(k)}, x_{2 n(k)}\right) .
$$

By taking the limit of (2.1) as $k \rightarrow \infty$ and using Lemma 1.9 we get

$$
\lim _{k \rightarrow \infty} p\left(x_{2 m(k)}, x_{2 n(k)-1}\right) \leqslant \varepsilon,
$$

and also

$$
p\left(x_{2 m(k)}, x_{2 n(k)}\right) \leqslant p\left(x_{2 m(k)}, x_{2 n(k)-1}\right)+p\left(x_{2 n(k)-1}, x_{2 n(k)}\right)-p\left(x_{2 n(k)-1}, x_{2 n(k)-1}\right) .
$$

By letting $k \rightarrow \infty$ in (2.3) and regarding Lemma 1.9 we get

$$
\varepsilon \leqslant \lim _{k \rightarrow \infty} p\left(x_{2 m(k)}, x_{2 n(k)-1}\right) .
$$

Therefore, from (2.2) and (2.4) we get that $\lim _{k \rightarrow \infty} p\left(x_{2 m(k)}, x_{2 n(k)-1}\right)=\varepsilon$.

The following is the main result of this paper.

Theorem 2.2. Let $(\mathrm{X}, \mathrm{p})$ be a complete partial metric space and $\mathrm{T}: \mathrm{X} \rightarrow \mathrm{X}$ be a self-mapping satisfying:

$$
\psi\left(p^{2}(T x, T y)\right) \leqslant \psi(M(x, y))-\varphi(M(x, y)), \quad \forall x, y \in X,
$$

where $\varphi \in \Phi, \psi \in \Psi$ and

$$
M(x, y)=\max \left\{\begin{array}{c}
p^{2}(x, y), \frac{p(x, T x) \cdot p(y, T y)}{1+p^{2}(x, y)}, \\
\frac{1}{2}[p(x, T y) \cdot p(y, T x)], \frac{p^{2}(y, T y)}{1+p^{2}(y, y)}
\end{array}\right\} .
$$

Then $\mathrm{T}$ has a unique fixed point, say $\mathrm{u}$, in $\mathrm{X}$ and $\lim _{\mathrm{n} \rightarrow \infty} \mathrm{T}^{\mathrm{n}}\left(\mathrm{x}_{0}\right)=\mathrm{u}$ for any arbitrary $\mathrm{x}_{0} \in \mathrm{X}$.

Proof. Let $x_{0} \in X$ be an arbitrary point and define the sequence $x_{n}=T^{n} x_{0}=T x_{n-1}$. Suppose that $x_{n}=x_{n+1}$ for some $n \in N$, then $x_{n}=x_{n+1}=T x_{n}$, so $x_{n}$ is a fixed point. From now on, we assume that $x_{n} \neq x_{n+1}$ for each $n \in N$. By using (2.5) for $x=x_{n-1}$ and $y=x_{n}$, we get that

$$
\begin{aligned}
\psi\left(p^{2}\left(x_{n}, x_{n+1}\right)\right) & =\psi\left(p^{2}\left(T x_{n-1}, T x_{n}\right)\right) \\
& \leqslant \psi\left(M\left(x_{n-1}, x_{n}\right)\right)-\varphi\left(M\left(x_{n-1}, x_{n}\right)\right),
\end{aligned}
$$

where

$$
M\left(x_{n-1}, x_{n}\right)=\max \left\{\begin{array}{c}
p^{2}\left(x_{n-1}, x_{n}\right), \frac{p\left(x_{n-1}, x_{n}\right) \cdot p\left(x_{n}, x_{n+1}\right)}{1+p^{2}\left(x_{n-1}, x_{n}\right)}, \\
\frac{1}{2}\left[p\left(x_{n-1}, x_{n+1}\right) \cdot p\left(x_{n}, x_{n}\right)\right], \frac{p^{2}\left(x_{n}, x_{n+1}\right)}{1+p^{2}\left(x_{n}, x_{n}\right)}
\end{array}\right\} .
$$

If $p\left(x_{n-1}, x_{n}\right) \leqslant p\left(x_{n}, x_{n+1}\right)$, then

$$
\frac{p\left(x_{n-1}, x_{n}\right) \cdot p\left(x_{n}, x_{n+1}\right)}{1+p^{2}\left(x_{n-1}, x_{n}\right)} \leqslant \frac{p^{2}\left(x_{n}, x_{n+1}\right)}{1+p^{2}\left(x_{n-1}, x_{n}\right)} \leqslant p^{2}\left(x_{n}, x_{n+1}\right) .
$$

Also, if $p\left(x_{n}, x_{n+1}\right) \leqslant p\left(x_{n-1}, x_{n}\right)$, then

$$
\frac{p\left(x_{n-1}, x_{n}\right) \cdot p\left(x_{n}, x_{n+1}\right)}{1+p^{2}\left(x_{n-1}, x_{n}\right)} \leqslant \frac{p^{2}\left(x_{n-1}, x_{n}\right)}{1+p^{2}\left(x_{n-1}, x_{n}\right)} \leqslant p^{2}\left(x_{n-1}, x_{n}\right) .
$$


Thus, we obtain

$$
\frac{p\left(x_{n-1}, x_{n}\right) \cdot p\left(x_{n}, x_{n+1}\right)}{1+p^{2}\left(x_{n-1}, x_{n}\right)} \leqslant \max \left\{p^{2}\left(x_{n}, x_{n+1}\right), p^{2}\left(x_{n-1}, x_{n}\right)\right\} .
$$

On the other hand, from (P4) and (P2) we have

$$
\begin{aligned}
\frac{1}{2}\left[p\left(x_{n-1}, x_{n+1}\right) \cdot p\left(x_{n}, x_{n}\right)\right] & \leqslant \frac{1}{2}\left[p\left(x_{n-1}, x_{n}\right) \cdot p\left(x_{n}, x_{n}\right)+p\left(x_{n}, x_{n+1}\right) \cdot p\left(x_{n}, x_{n}\right)-p^{2}\left(x_{n}, x_{n}\right)\right] \\
& \leqslant \frac{1}{2}\left[p^{2}\left(x_{n-1}, x_{n}\right)+p^{2}\left(x_{n}, x_{n+1}\right)-p^{2}\left(x_{n}, x_{n}\right)\right] \\
& \leqslant \frac{1}{2}\left[p^{2}\left(x_{n-1}, x_{n}\right)+p^{2}\left(x_{n}, x_{n+1}\right)\right] \\
& \leqslant \max \left\{p^{2}\left(x_{n-1}, x_{n}\right), p^{2}\left(x_{n}, x_{n+1}\right)\right\} .
\end{aligned}
$$

Combining the observed results in (2.7) and (2.8), we find that

$$
M\left(x_{n-1}, x_{n}\right) \leqslant \max \left\{p^{2}\left(x_{n-1}, x_{n}\right), p^{2}\left(x_{n}, x_{n+1}\right)\right\} .
$$

Therefore, by the help of (2.9), inequality (2.6) becomes,

$$
\begin{aligned}
\psi\left(p^{2}\left(x_{n}, x_{n+1}\right)\right)= & \psi\left(p^{2}\left(T x_{n-1}, T x_{n}\right)\right) \\
\leqslant & \psi\left(\max \left\{p^{2}\left(x_{n-1}, x_{n}\right), p^{2}\left(x_{n}, x_{n+1}\right)\right\}\right) \\
& -\varphi\left(\max \left\{p^{2}\left(x_{n-1}, x_{n}\right), p^{2}\left(x_{n}, x_{n+1}\right)\right\}\right),
\end{aligned}
$$

which implies that

$$
\psi\left(p^{2}\left(x_{n}, x_{n+1}\right)\right)<\psi\left(\max \left\{p^{2}\left(x_{n-1}, x_{n}\right), p^{2}\left(x_{n}, x_{n+1}\right)\right\}\right) .
$$

If $\max \left\{p^{2}\left(x_{n-1}, x_{n}\right), p^{2}\left(x_{n}, x_{n+1}\right)\right\}=p^{2}\left(x_{n}, x_{n+1}\right)$, then we have

$$
\psi\left(p^{2}\left(x_{n}, x_{n+1}\right)\right)<\psi\left(p^{2}\left(x_{n}, x_{n+1}\right)\right),
$$

a contradiction. Hence, the inequality (2.11) yields that

$$
\psi\left(p^{2}\left(x_{n}, x_{n+1}\right)\right)<\psi\left(p^{2}\left(x_{n-1}, x_{n}\right)\right) .
$$

Since $\psi$ is non-decreasing, the inequality above turns into

$$
p^{2}\left(x_{n}, x_{n+1}\right) \leqslant p^{2}\left(x_{n-1}, x_{n}\right),
$$

that is

$$
p\left(x_{n}, x_{n+1}\right) \leqslant p\left(x_{n-1}, x_{n}\right) .
$$

Therefore, the sequence $\left\{p\left(x_{n}, x_{n+1}\right)\right\}$ is decreasing sequence and bounded below by 0 . So,

$$
\lim _{n \rightarrow \infty} p\left(x_{n}, x_{n+1}\right)=r \geqslant 0 .
$$

Now we show that $r=0$. Assume $r>0$. Taking limit as $n \rightarrow \infty$ for (2.10) we get

$$
\begin{aligned}
\psi\left(r^{2}\right) & \leqslant \psi\left(\max \left\{r^{2}, r^{2}\right\}\right)-\varphi\left(\max \left\{r^{2}, r^{2}\right\}\right) \\
& <\psi\left(r^{2}\right)
\end{aligned}
$$

a contradiction. Thus, we conclude that $r=0$ and so,

$$
\lim _{n \rightarrow \infty} p\left(x_{n}, x_{n+1}\right)=0 .
$$


As a next step, we shall prove that $\left\{x_{n}\right\}$ is a Cauchy sequence in the context of partial metric space $(X, p)$. Suppose, on the contrary, that the sequence $\left\{x_{n}\right\}$ is not Cauchy. Then, by Lemma 1.10, the subsequence $\left\{x_{2 n}\right\}$ is not Cauchy, either. By Lemma 1.9 there exist $\varepsilon>0$ and two sequences $\{m(k)\}$ and $\{n(k)\}$ of positive integers such that $n(k)>m(k)>k$ and

$$
\lim _{k \rightarrow \infty} p\left(x_{2 n(k)}, x_{2 m(k)}\right)=\lim _{k \rightarrow \infty} p\left(x_{2 m(k)-1}, x_{2 n(k)}\right)=\lim _{k \rightarrow \infty} p\left(x_{2 n(k)-1}, x_{2 m(k)}\right)=\varepsilon .
$$

Now, we have

$$
\begin{aligned}
\psi\left(p^{2}\left(x_{2 n(k)}, x_{2 m(k)}\right)\right) & =\psi\left(p^{2}\left(T x_{2 n(k)-1}, T x_{2 m(k)-1}\right)\right) \\
& \leqslant \psi\left(M\left(x_{2 n(k)-1}, x_{2 m(k)-1}\right)\right)-\varphi\left(M\left(x_{2 n(k)-1}, x_{2 m(k)-1}\right)\right)
\end{aligned}
$$

where

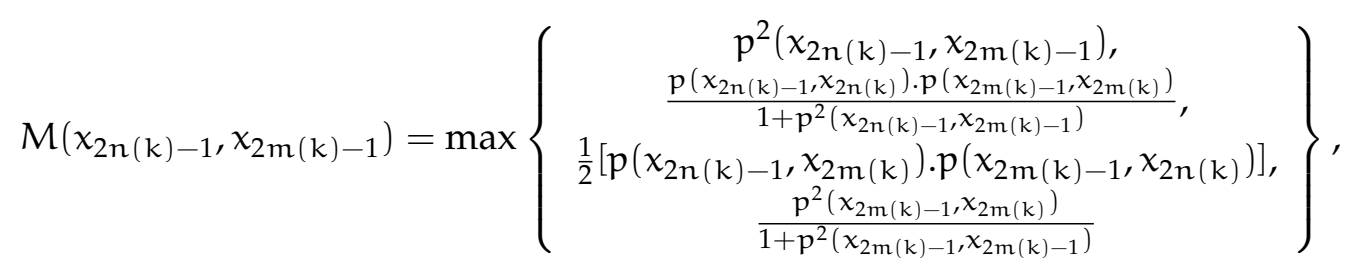

but,

$$
\begin{aligned}
0 \leqslant p^{2}\left(x_{2 n(k)-1}, x_{2 m(k)-1}\right) \leqslant & {\left[p\left(x_{2 n(k)-1}, x_{2 n(k)}\right)\right.} \\
& \left.+p\left(x_{2 n(k)}, x_{2 m(k)-1}\right)-p\left(x_{2 n(k)}, x_{2 n(k)}\right)\right]^{2} .
\end{aligned}
$$

Hence, we have

$$
\begin{aligned}
& \max \left\{\begin{array}{c}
\frac{p\left(x_{2 n(k)-1}, x_{2 n(k)}\right) \cdot p\left(x_{2 m(k)-1}, x_{2 m(k)}\right)}{1+p^{2}\left(x_{2 n(k)-1}, x_{2 m(k)-1}\right)} \\
\frac{1}{2}\left[p \left(x_{\left.\left.2 n(k)-1, x_{2 m(k)}\right) \cdot p\left(x_{2 m(k)-1}, x_{2 n(k)}\right)\right]}\right.\right.
\end{array}\right\} \\
& \leqslant M\left(x_{2 \mathfrak{n}(k)-1}, x_{2 m(k)-1}\right) \\
& \leqslant \max \left\{\begin{array}{c}
{\left[p\left(x_{2 n(k)-1}, x_{2 n(k)}\right)+p\left(x_{2 n(k)}, x_{2 m(k)-1}\right)-p\left(x_{2 n(k)}, x_{2 n(k)}\right)\right]^{2},} \\
\frac{p\left(x_{2 n(k)-1}, x_{2 n(k)}\right) \cdot p\left(x_{2 m(k)-1}, x_{2 m(k)}\right)}{1+p^{2}\left(x_{2 n}(k)-1, x_{2 m(k)-1}\right)}, \\
\frac{1}{2}\left[p\left(x_{2 n(k)-1}, x_{2 m(k)}\right) \cdot p\left(x_{2 m(k)-1}, x_{2 n(k)}\right)\right], \\
\frac{p^{2}\left(x_{2 m(k)-1}, x_{2 m(k)}\right)}{1+p^{2}\left(x_{2 m(k)-1}, x_{2 m(k)-1}\right)}
\end{array}\right\} .
\end{aligned}
$$

Letting $k \rightarrow \infty$ in (2.14) together with (2.12), (2.13), Lemma 2.1, and (P2) we get

$$
\begin{aligned}
\psi\left(\varepsilon^{2}\right) & \leqslant \psi\left(\max \left\{\varepsilon^{2}, 0, \frac{\varepsilon^{2}}{2}, 0\right\}\right)-\varphi\left(\lim _{k \rightarrow \infty} M\left(x_{2 n(k)-1}, x_{2 m(k)-1}\right)\right) \\
& =\psi\left(\varepsilon^{2}\right)-\varphi\left(\lim _{k \rightarrow \infty} M\left(x_{2 n(k)-1}, x_{2 m(k)-1}\right)\right) .
\end{aligned}
$$

From (2.15), $\lim _{k \rightarrow \infty} M\left(x_{2 n(k)-1}, x_{2 m(k)-1}\right) \geqslant \max \left\{0, \frac{\varepsilon^{2}}{2}, 0\right\}=\frac{\varepsilon^{2}}{2}>0$, and so from properties of $\varphi$ we conclude that $\varphi\left(\lim _{k \rightarrow \infty} M\left(x_{2 n(k)-1}, x_{2 m(k)-1}\right)\right)>0$. Thus $(2.16)$ becomes $\psi\left(\varepsilon^{2}\right)<\psi\left(\varepsilon^{2}\right)$, a contradiction. So, we deduce that $\left\{x_{2 n}\right\}$ is a Cauchy sequence. Hence, by Lemma $1.10\left\{x_{n}\right\}$ is a Cauchy sequence. So, there exists $u \in X$ such that $\lim _{n \rightarrow \infty} x_{n}=u$. From (P2) and Lemma 1.11 that $0=\lim _{n \rightarrow \infty} p\left(x_{n}, u\right)=p(u, u)$ and hence,

$$
\lim _{n \rightarrow \infty} p^{2}\left(x_{n}, u\right)=0
$$


Next, we shall show that $u \in X$ is a fixed point of $T$. From (2.5) we have

$$
\begin{aligned}
\psi\left(p^{2}\left(x_{n+1}, T u\right)\right) & =\psi\left(p^{2}\left(T x_{n}, T u\right)\right) \\
& \leqslant \psi\left(M\left(x_{n}, u\right)\right)-\varphi\left(M\left(x_{n}, u\right)\right),
\end{aligned}
$$

where

$$
M\left(x_{n}, u\right)=\max \left\{\begin{array}{c}
p^{2}\left(x_{n}, u\right), \frac{p\left(x_{n}, T u\right) \cdot p(u, T u)}{1+p^{2}\left(x_{n}, u\right)}, \\
\frac{1}{2}\left[p\left(x_{n}, T u\right) \cdot p\left(u, x_{n+1}\right)\right], \frac{p^{2}(u, T u)}{1+p^{2}(u, u)}
\end{array}\right\} .
$$

By taking limit for $M\left(x_{n}, u\right)$ as $n \rightarrow \infty$ and using (2.17) and Lemma 1.5, then equation (2.18) becomes,

$$
\psi\left(p^{2}(u, T u)\right) \leqslant \psi\left(p^{2}(u, T u)\right)-\varphi\left(p^{2}(u, T u)\right) .
$$

Consequently, $\varphi\left(p^{2}(u, T u)\right)=0$, so $p^{2}(u, T u)=0$ which implies $p(u, T u)=0$ and $u=T u$.

Now, we shall prove that the obtained fixed point is unique. Suppose, on the contrary, that there exists another fixed point $v \in X$ with $v \neq u$. Then, we observe

$$
\begin{aligned}
\psi\left(p^{2}(v, u)\right) & =\psi\left(p^{2}(T v, T u)\right) \\
& \leqslant \psi(M(v, u))-\varphi(M(v, u)),
\end{aligned}
$$

where

$$
M(u, v)=\max \left\{\begin{array}{c}
p^{2}(u, v), \frac{p(u, T u) \cdot p(v, T v)}{1+p^{2}(u, v)}, \\
\frac{1}{2}[p(u, T v) \cdot p(v, T u)], \frac{p^{2}(v, T v)}{1+p^{2}(v, v)}
\end{array}\right\}
$$

Hence, we find

$$
\begin{aligned}
\psi\left(p^{2}(u, v)\right) \leqslant & \psi\left(\max \left\{p^{2}(u, v), 0, \frac{p^{2}(u, v)}{2}, \frac{p^{2}(v, v)}{1+p^{2}(v, v)}\right\}\right) \\
& -\varphi\left(\max \left\{p^{2}(u, v), 0, \frac{p^{2}(u, v)}{2}, \frac{p^{2}(v, v)}{1+p^{2}(v, v)}\right\}\right),
\end{aligned}
$$

but $\frac{p^{2}(v, v)}{1+p^{2}(v, v)} \leqslant \frac{p^{2}(u, v)}{1+p^{2}(v, v)} \leqslant p^{2}(u, v)$. Thus (2.19) becomes

$$
\psi\left(p^{2}(u, v)\right) \leqslant \psi\left(p^{2}(u, v)\right)-\varphi\left(p^{2}(u, v)\right),
$$

which yields

$$
\psi\left(p^{2}(u, v)\right)<\psi\left(p^{2}(u, v)\right),
$$

a contradiction. This completes the proof.

Theorem 2.3. Let $(\mathrm{X}, \mathrm{p})$ be a complete partial metric space and $\mathrm{T}: \mathrm{X} \rightarrow \mathrm{X}$ be a self mapping satisfying for some $n \in \mathbb{N}$

$$
\psi\left(p^{2}\left(T^{n} x, T^{n} y\right)\right) \leqslant \psi(M(x, y))-\varphi(M(x, y)), \quad \forall x, y \in X,
$$

where $\varphi \in \Phi, \psi \in \Psi$ and

$$
M(x, y)=\max \left\{\begin{array}{c}
p^{2}(x, y), \frac{p\left(x, T^{n} x\right) \cdot p\left(y, T^{n} y\right)}{1+p^{2}(x, y)} \\
\frac{1}{2}\left[p\left(x, T^{n} y\right) \cdot p\left(y, T^{n} x\right)\right], \frac{p^{2}\left(y, T^{n} y\right)}{1+p^{2}(y, y)}
\end{array}\right\} .
$$

Then $\mathrm{T}$ has a unique fixed point, say $\mathrm{u}$, in $\mathrm{X}$ and $\lim _{\mathrm{k} \rightarrow \infty} \mathrm{T}^{\mathrm{k}}\left(\mathrm{x}_{0}\right)=\mathrm{u}$ for any arbitrary $\mathrm{x}_{0} \in \mathrm{X}$.

Proof. By following the proof of Theorem 2.2, we conclude that the mapping $\mathrm{T}^{\mathrm{n}}$ has a unique fixed point, say $u$, that is $T^{n} \mathfrak{u}=u$. Thus, we have $T u=T\left(T^{n} u\right)=T^{n+1} \mathfrak{u}=T^{n}(T u)$. As a result, $T u$ is a fixed 
point of $T^{n}$. On account of Lemma 1.13, uniqueness yields that $u=T u$. From Theorem 2.2 we have $\lim _{m \rightarrow \infty} T^{m n}\left(x_{0}\right)=u$, thus

$$
\begin{aligned}
\lim _{k \rightarrow \infty} T^{k}\left(x_{0}\right) & =\lim _{m \rightarrow \infty} T^{m n+r} x_{0} \\
& =\lim _{m \rightarrow \infty} T^{m n}\left(T^{r} x_{0}\right) \\
& =\lim _{m \rightarrow \infty} T^{m n}\left(x^{*}\right)=u,
\end{aligned}
$$

where $0 \leqslant r<n$ and $x^{*}=T^{r} x_{0} \in X$.

Definition 2.4 ([19]). We say that a map $T$ has the property $P$ if $F(T)=F\left(T^{n}\right)$ for each $n \in \mathbb{N}$, where $F(T)$ denotes the set of all fixed points of a self-mapping $T$.

Theorem 2.5. Let $(\mathrm{X}, \mathrm{p})$ be a complete partial metric space and $\mathrm{T}: \mathrm{X} \rightarrow \mathrm{X}$ be a self-mapping satisfying:

$$
\psi\left(p^{2}(T x, T y)\right) \leqslant \psi(M(x, y))-\varphi(M(x, y)), \quad \forall x, y \in X,
$$

where $\varphi \in \Phi, \psi \in \Psi$ and

$$
M(x, y)=\max \left\{\begin{array}{c}
p^{2}(x, y), \frac{p(x, T x) \cdot p(y, T y)}{1+p^{2}(x, y)} \\
\frac{1}{2}[p(x, T y) \cdot p(y, T x)], \frac{p^{2}(y, T y)}{1+p^{2}(y, y)}
\end{array}\right\}
$$

Then, $\mathrm{T}$ has the property $\mathrm{P}$.

Proof. Let $n \in \mathbb{N}$ and suppose $u \in F\left(T^{n}\right)$. Then for any integers $0<i, j<n$, we have

$$
\psi\left(p^{2}\left(T^{i} \mathfrak{u}, T^{j} \mathfrak{u}\right)\right) \leqslant \psi\left(M\left(T^{i-1} \mathfrak{u}, T^{j-1} \mathfrak{u}\right)\right)-\varphi\left(M\left(T^{i-1} \mathfrak{u}, T^{j-1} \mathfrak{u}\right)\right),
$$

where,

$$
M\left(T^{i-1} \mathfrak{u}, T^{j-1} \mathfrak{u}\right)=\max \left\{\begin{array}{c}
p^{2}\left(T^{i-1} \mathfrak{u}, T^{j-1} \mathfrak{u}\right), \frac{p\left(T^{i-1} \mathfrak{u}, T^{i} \mathfrak{u}\right) \cdot p\left(T^{j-1} \mathfrak{u}, T^{j} \mathfrak{u}\right)}{1+p^{2}\left(T^{i-1} \mathfrak{u}, T^{j-1} \mathfrak{u}\right)}, \\
\frac{1}{2}\left[p\left(T^{i-1} \mathfrak{u}, T^{j} \mathfrak{u}\right) \cdot p\left(T^{j-1} \mathfrak{u}, T^{i} u\right)\right], \frac{p^{2}\left(T^{j-1} \mathfrak{u}, T^{j} \mathfrak{u}\right)}{1+p^{2}\left(T^{j-1} \mathfrak{u}, T^{j-1} \mathfrak{u}\right)}
\end{array}\right\} .
$$

For the sake of simplicity, we define

$$
\Delta=\max _{0 \leqslant i, j<n}\left\{p^{2}\left(T^{i} u, T^{j} u\right)\right\}
$$

Now, we assume that $M\left(T^{i-1} \mathfrak{u}, T^{j-1} \mathfrak{u}\right) \neq 0$ for all $0<i, j \leqslant n$.

$$
\begin{aligned}
\psi\left(p^{2}\left(T^{i} \mathfrak{u}, T^{j} \mathfrak{u}\right)\right) & \leqslant \psi\left(M\left(T^{i-1} \mathfrak{u}, T^{j-1} \mathfrak{u}\right)\right)-\varphi\left(M\left(T^{i-1} \mathfrak{u}, T^{j-1} \mathfrak{u}\right)\right) \\
& \leqslant \psi(\Delta)-\varphi\left(M\left(T^{i-1} \mathfrak{u}, T^{j-1} \mathfrak{u}\right)\right) \\
& <\psi(\Delta) .
\end{aligned}
$$

By taking maximum of (2.20) for $0 \leqslant i, j<n$, we get that $\psi(\Delta)<\psi(\Delta)$ which is a contradiction. Thus there exist $i_{0}, j_{0}$ such that $M\left(T^{i_{0}-1} \mathfrak{u}, T^{j_{0}-1} \mathfrak{u}\right)=0$, then we have $p^{2}\left(T^{j_{0}-1} \mathfrak{u}, T^{j_{0}} \mathfrak{u}\right)=0$. Consequently, we get $p\left(T^{j_{0}-1} \mathfrak{u}, T^{j_{0}} \mathfrak{u}\right)=0$. Combining the observations above with the axioms (P1) and (P2), we get $T^{j_{0}-1} \mathfrak{u}=T^{j_{0}} \mathfrak{u}$. Therefore

$$
T^{n} u=T^{n-j_{0}+1}\left(T^{j_{0}-1} \mathfrak{u}\right)=T^{n-j_{0}+1}\left(T^{j_{0}} u\right)=T^{n+1} u .
$$

Thus, $T\left(T^{n} u\right)=T^{n+1}=T^{n} u$. Since, $u=T^{n} u$, we conclude that $T u=u$. Hence $u$ is a fixed point of $T$. Therefore, $F(T)=F\left(T^{n}\right)$, for all $n \in \mathbb{N}$. 


\section{Some consequences}

In Theorem 2.2, by taking $\psi(t)=t$ and $\varphi(t)=(1-\alpha) t$ such that $\alpha \in(0,1)$ we get the following corollary.

Corollary 3.1. Let $(\mathrm{X}, \mathrm{p})$ be a complete partial metric space and $\mathrm{T}: \mathrm{X} \rightarrow \mathrm{X}$ be a self-mapping satisfying

$$
p^{2}(T x, T y) \leqslant \alpha M(x, y), \quad \forall x, y \in X,
$$

where

$$
M(x, y)=\max \left\{\begin{array}{c}
p^{2}(x, y), \frac{p(x, T x) \cdot p(y, T y)}{1+p^{2}(x, y)}, \\
\frac{1}{2}[p(x, T y) \cdot p(y, T x)], \frac{p^{2}(y, T y)}{1+p^{2}(y, y)}
\end{array}\right\} .
$$

Then $\mathrm{T}$ has a unique fixed point, say $\mathrm{u}$, in $\mathrm{X}$ and $\lim _{\mathrm{n} \rightarrow \infty} \mathrm{T}^{\mathrm{n}}\left(\mathrm{x}_{0}\right)=\mathrm{u}$ for any arbitrary $\mathrm{x}_{0} \in \mathrm{X}$.

Corollary 3.2. Let $(X, p)$ be a complete partial metric space and $\mathrm{T}: \mathrm{X} \rightarrow \mathrm{X}$ be a self-mapping satisfying

$$
p^{2}(T x, T y) \leqslant M(x, y), \quad \forall x, y \in X
$$

where

$$
M(x, y)=a_{1} p^{2}(x, y)+a_{2} \frac{p(x, T x) \cdot p(y, T y)}{1+p^{2}(x, y)}+a_{3}[p(x, T y) \cdot p(y, T x)]+a_{4} \frac{p^{2}(y, T y)}{1+p^{2}(y, y)}
$$

and $\mathrm{a}_{1}+\mathrm{a}_{2}+2 \mathrm{a}_{3}+\mathrm{a}_{4}<1$. Then $\mathrm{T}$ has a unique fixed point, say $\mathrm{u}$, in $\mathrm{X}$ and $\lim _{\mathrm{n} \rightarrow \infty} \mathrm{T}^{\mathrm{n}}\left(\mathrm{x}_{0}\right)=\mathrm{u}$ for any arbitrary $x_{0} \in X$.

By taking $a_{2}=a_{3}=a_{4}=0$ in the above corollary we get the following result ([38, Theorem 5.3]).

Corollary 3.3 ([38], Theorem 5.3). Let $(X, p)$ be a complete partial metric space and $\mathrm{T}: \mathrm{X} \rightarrow \mathrm{X}$ be a self-mapping satisfying

$$
p(T x, T y) \leqslant \beta p(x, y), \quad \forall x, y \in X, \quad \beta \in[0,1) .
$$

Then $T$ has a unique fixed point, say $u$, in $X$ and $\lim _{n \rightarrow \infty} T^{n}\left(x_{0}\right)=u$ for any arbitrary $x_{0} \in X$.

Denote by $\Lambda$ the set of functions $\lambda:[0, \infty) \rightarrow[0, \infty)$ satisfying the following hypotheses:

1. $\lambda$ is a Lebesgue integral mapping on each compact subset of $[0, \infty)$.

2. For every $\varepsilon>0$, we have $\int_{0}^{\varepsilon} \lambda(s) \mathrm{d} s>0$.

Corollary 3.4. Let $(\mathrm{X}, \mathrm{p})$ be a complete partial metric space and $\mathrm{T}: \mathrm{X} \rightarrow \mathrm{X}$ be a self-mapping satisfying

$$
\int_{0}^{p^{2}(T x, T y)} \lambda(s) d s \leqslant \int_{0}^{M(x, y)} \lambda(s) d s-\int_{0}^{M(x, y)} \mu(s) d s, \quad \forall x, y \in X,
$$

where $\lambda, \mu \in \Lambda$ and

$$
M(x, y)=\max \left\{\begin{array}{c}
p^{2}(x, y), \frac{p(x, T x) \cdot p\left(y, T^{n} y\right)}{1+p^{2}(x, y)}, \\
\frac{1}{2}[p(x, T y) \cdot p(y, T x)], \frac{p^{2}(y, T y)}{1+p^{2}(y, y)}
\end{array}\right\} .
$$

Then $\mathrm{T}$ has a unique fixed point, say $\mathrm{u}$, in $\mathrm{X}$.

Proof. It is an easy matter to see that the mappings $\psi, \varphi:[0, \infty) \rightarrow[0, \infty)$ defined by $\psi(t)=\int_{0}^{t} \lambda(s) d s$ and $\varphi(t)=\int_{0}^{t} \mu(s) d s$ are (altering) and strong-altering distance functions respectively. Therefore, the rest of the proof follows from the proof of Theorem 2.2.

Corollary 3.5. Let $(\mathrm{X}, \mathrm{p})$ be a complete partial metric space and $\mathrm{T}: \mathrm{X} \rightarrow \mathrm{X}$ be a self-mapping satisfying

$$
\psi\left(p^{2}\left(T x, T^{2} x\right)\right) \leqslant \psi(M(x, T x))-\varphi(M(x, T x)), \quad \forall x \in X,
$$

where $\varphi \in \Phi, \psi \in \Psi$ and 


$$
M(x, T x)=\max \left\{\begin{array}{c}
p^{2}(x, T x), \frac{p(x, T x) \cdot p\left(T x, T^{2} x\right)}{1+p^{2}(x, T x)}, \\
\frac{1}{2}\left[p\left(x, T^{2} x\right) \cdot p(T x, T x)\right] \frac{p^{2}\left(T x, T^{2} x\right)}{1+p^{2}(T x, T x)}
\end{array}\right\} .
$$

Then, $\mathrm{T}$ has a unique fixed point, say $\mathrm{u}$, in $\mathrm{X}$ and $\lim _{\mathrm{n} \rightarrow \infty} \mathrm{T}^{\mathrm{n}}\left(\mathrm{x}_{0}\right)=\mathrm{u}$ for any arbitrary initial point $\mathrm{x}_{0} \in \mathrm{X}$.

Proof. The proof follows from Theorem 2.2 by taking $y=T x$.

Now, we give an example to support Theorem 2.2.

Example 3.6. Let $X=[0, \infty)$, and $(X, p)$ be a partial metric space which is defined by $p(x, y)=\max \{x, y\}$ for all $x, y \in X$. Let $T$ be a self-mapping defined by

$$
\mathrm{T} x=\frac{4 x}{5}, \quad \forall x \in[0, \infty),
$$

and $\psi(t)=t^{2}, \varphi(t)=\frac{2}{5} t^{2}$ for all $t \in[0, \infty)$. Let $x, y \in X$. Without loss of generality assume that $x<y$, then

$$
\begin{aligned}
& p^{2}(T x, T y)=\left(\max \left\{\frac{4 x}{5}, \frac{4 y}{5}\right\}\right)^{2}=\frac{16 y^{2}}{25}, \\
& p^{2}(x, y)=(\max \{x, y\})^{2}=y^{2}, \\
& p(x, T x)=\max \left\{x, \frac{4 x}{5}\right\}=x, \\
& p(y, T y)=\max \left\{y, \frac{4 y}{5}\right\}=y, \\
& p(y, T x)=\max \left\{y, \frac{4 x}{5}\right\}=y, \\
& p(x, T y)=\max \left\{x, \frac{4 y}{5}\right\} .
\end{aligned}
$$

Now to find $M(x, y)$ we split our work into two cases:

Case 1: If $5 x>4 y$, then $p(x, T y)=x$, so

$$
M(x, y)=\max \left\{y^{2}, \frac{x y}{1+y^{2}}, \frac{x y}{2}, \frac{y^{2}}{1+y^{2}}\right\}=y^{2} .
$$

Case 2: If $5 x \leqslant 4 y$, then $p(x, T y)=\frac{4 y}{5}$, so

$$
M(x, y)=\max \left\{y^{2}, \frac{x y}{1+y^{2}}, \frac{4 y^{2}}{10}, \frac{y^{2}}{1+y^{2}}\right\}=y^{2} .
$$

Hence we deduce that

$$
\begin{aligned}
\psi\left(p^{2}(T x, T y)\right) & =\psi\left(\frac{16 y^{2}}{25}\right)=\frac{256}{625} y^{4} \\
& \leqslant \frac{3}{5} y^{4} \\
& =y^{4}-\left(\frac{2}{5} y^{4}\right) \\
& =\psi\left(y^{2}\right)-\varphi\left(y^{2}\right) \\
& =\psi(M(x, y))-\varphi(M(x, y)) .
\end{aligned}
$$

Therefore, all conditions of Theorem 2.2 are satisfied and $x=0$ is the unique fixed point of $T$. 


\section{Application to integral equation}

In this section, we will use Theorem 2.2 to show that there is a solution to the following integral equation:

$$
x(t)=h(x(t))+\int_{0}^{t} m(t, s) H(s, x(s)) d s, \quad t \in[0,1],
$$

where,

1. $h(t):[0,1] \rightarrow R$ is a continuous function.

2. $\mathrm{m}(\mathrm{t}, \mathrm{s}):[0,1] \times[0,1] \rightarrow[0, \infty)$ is a continuous functions.

3. $H(t, s):[0,1] \times R \rightarrow R$ is a continuous function.

Let $X=C([0,1])$ be the set of all real continuous functions on $[0,1]$, endowed with the partial metric

$$
p(u, v)=\max \left\{\sup _{t \in[0,1]}|u(t)|, \sup _{t \in[0,1]}|v(t)|\right\}, \quad \forall u, v \in X .
$$

Clearly, $(X, p)$ is a complete partial metric space.

Theorem 4.1. The integral equation (4.1) has a solution $\mathrm{u} \in \mathrm{C}([0,1])$ if the following conditions hold:

1. $\sup _{t \in[0,1]} m(t, s) \leqslant \frac{1}{\sqrt{5}}$.

2. $|H(s, t)| \leqslant|t|$.

3. $|h(t)| \leqslant \frac{1}{\sqrt{5}}|t|$.

Proof. Define mapping $\mathrm{T}: \mathrm{X} \rightarrow \mathrm{X}$ by

$$
T x(t)=h(x(t))+\int_{0}^{t} m(t, s) H(s, x(s)) d s, \quad t \in[0,1] .
$$

We now prove condition (2.5) of Theorem 2.2 is satisfied. Let $x(t), y(t) \in X$. Then, for all $t \in[0,1]$, we have

$$
\begin{aligned}
|T(x(t))| & \leqslant|h(x(t))|+\left|\int_{0}^{t} m(t, s) H(s, x(s)) d s\right| \\
& \leqslant|h(x(t))|+\int_{0}^{t}|m(t, s)||H(s, x(s))| d s \\
& \leqslant|h(x(t))|+\int_{0}^{t} \frac{1}{\sqrt{5}}|H(s, x(s))| d s \\
& \leqslant \frac{1}{\sqrt{5}} \sup _{t \in[0,1]}|x(t)|+\int_{0}^{t} \frac{1}{\sqrt{5}} \sup _{s \in[0,1]}|x(s)| d s \\
& \leqslant \frac{1}{\sqrt{5}} \sup _{t \in[0,1]} \mid x\left(t\left|+\int_{0}^{1} \frac{1}{\sqrt{5}} \sup _{s \in[0,1]}\right| x(s) \mid d s\right. \\
& =\frac{2}{\sqrt{5}} \sup _{t \in[0,1]}|x(t)| .
\end{aligned}
$$

Similarly,

$$
|T(y(t))| \leqslant \frac{2}{\sqrt{5}} \sup _{t \in[0,1]}|y(t)| .
$$

Hence,

$$
p(T(x(t)), T(y(t))) \leqslant \frac{2}{\sqrt{5}} p(x(t), y(t))
$$

which implies, 


$$
\begin{aligned}
p^{2}(T(x(t)), T(y(t))) & \leqslant \frac{4}{5} p^{2}(x(t), y(t)) \\
& \leqslant \frac{4}{5} M(x, y) \\
& =\psi(M(x, y))-\varphi(M(x, y))
\end{aligned}
$$

Therefore, $\psi\left(p^{2}(T(x(t)), T(y(t)))\right) \leqslant \psi(M(x, y))-\varphi(M(x, y))$ where $\psi(t)=t$ and $\varphi(t)=\frac{t}{5}$. Hence, all conditions of Theorem 2.2 hold and the mapping $T$ has a fixed point $u(t) \in C([0,1])$ which is a solution to the equation (4.1).

Example 4.2. The equation

$$
\frac{4}{5} x(t)=\frac{1}{2 \sqrt{5}} \int_{0}^{t} t s x(s) d s, \quad t \in[0,1]
$$

has a solution.

Proof. Let $\mathrm{m}(\mathrm{t}, \mathrm{s})=\frac{\mathrm{ts}}{\sqrt{5}}, \mathrm{H}(\mathrm{s}, \mathrm{t})=\frac{1}{2} \mathrm{t}$ and $\mathrm{h}(\mathrm{t})=\frac{\mathrm{t}}{5}$. Then all conditions of Theorem 4.1 are satisfied and so the equation has a solution.

\section{Conclusion}

As it is known well, a metric space satisfies all conditions of the notion of partial metric space. But, the converse is false. Hence, the observed fixed point results of this paper, in the setting of partial metric spaces generalize and cover all corresponding results in the context of metric spaces. In particular, we re-state all results of this paper in the setting of metric spaces as consequences. We avoid the list all such consequences to optimize the length of the paper.

\section{References}

[1] T. Abdeljawad, E. Karapınar, K. Taş, Existence and uniqueness of a common fixed point on partial metric spaces, Appl. Math. Lett., 24 (2011), 1900-1904. 1, 1.5, 1.6

[2] M. Akram, W. Shamaila, Fixed point results in partial metric spaces using generalized weak contractive conditions, J. Math. Comput. Sci., 12 (2014), 85-98.

[3] H. Aydi, M. Abbas, C. Vetro, Partial Hausdorff metric and Nadler's fixed point theorem on partial metric spaces, Topology Appl., 159 (2012), 3234-3242.

[4] H. Aydi, M. Abbas, C. Vetro, Common fixed points for multivalued generalized contractions on partial metric spaces, Rev. R. Acad. Cienc. Exactas Fís. Nat. Ser. A Math. RACSAM, 108 (2014), 483-501.

[5] H. Aydi, S. Hadj Amor, E. Karapınar, Berinde-type generalized contractions on partial metric spaces, Abstr. Appl. Anal., 2013 (2013), 10 pages.

[6] H. Aydi, M. Jellali, E. Karapınar, Common fixed points for generalized $\alpha$-implicit contractions in partial metric spaces: consequences and application, Rev. R. Acad. Cienc. Exactas Fís. Nat. Ser. A Math. RACSAM, 109 (2015), 367-384.

[7] H. Aydi, E. Karapınar, A Meir-Keeler common type fixed point theorem on partial metric spaces, Fixed Point Theory Appl., 2012 (2012), 10 pages.

[8] H. Aydi, E. Karapınar, New Meir-Keeler type tripled fixed-point theorems on ordered partial metric spaces, Math. Probl. Eng., 2012 (2012), 17 pages.

[9] H. Aydi, E. Karapınar, P. Kumam, A note on 'Modified proof of Caristi's fixed point theorem on partial metric spaces, Journal of Inequalities and Applications 2013, 2013:210', J. Inequal. Appl., 2013 (2013), 3 pages.

[10] H. Aydi, E. Karapınar, S. Rezapour, A generalized Meir-Keeler-type contraction on partial metric spaces, Abstr. Appl. Anal., 2012 (2012), 10 pages.

[11] H. Aydi, E. Karapınar, W. Shatanawi, Coupled fixed point results for $(\psi, \phi)$-weakly contractive condition in ordered partial metric spaces, Comput. Math. Appl., 62 (2011), 4449-4460.

[12] H. Aydi, E. Karapınar, C. Vetro, On Ekeland's variational principle in partial metric spaces, Appl. Math. Inf. Sci., 9 (2015), 257-262.

[13] H. Aydi, C. Vetro, W. Sintunavarat, P. Kumam, Coincidence and fixed points for contractions and cyclical contractions in partial metric spaces, Fixed Point Theory Appl., 2012 (2012), 18 pages. 
[14] K. P. Chi, E. Karapınar, T. D. Thanh, A generalized contraction principle in partial metric spaces, Math. Comput. Modelling, 55 (2012), 1673-1681.

[15] K. P. Chi, E. Karapınar, T. D. Thanh, On the fixed point theorems in generalized weakly contractive mappings on partial metric spaces, Bull. Iranian Math. Soc., 39 (2013), 369-381.

[16] İ. M. Erhan, E. Karapınar, D. Türkoğlu, Different types Meir-Keeler contractions on partial metric spaces, J. Comput. Anal. Appl., 14 (2012), 1000-1005.

[17] S. Gülyaz, E. Karapınar, A coupled fixed point result in partially ordered partial metric spaces through implicit function, Hacet. J. Math. Stat., 42 (2013), 347-357.

[18] D. Ilić, V. Pavlović, V. Rakočević, Some new extensions of Banach's contraction principle to partial metric space, Appl. Math. Lett., 24 (2011), 1326-1330. 1

[19] G. S. Jeong, B. E. Rhoades, More maps for which $\mathrm{F}(\mathrm{T})=\mathrm{F}\left(\mathrm{T}^{\mathrm{n}}\right)$, Demonstratio Math., 40 (2007), 671-680. 2.4

[20] M. Jleli, E. Karapınar, B. Samet, Further remarks on fixed-point theorems in the context of partial metric spaces, Abstr. Appl. Anal., 2013 (2013), 6 pages. 1

[21] E. Karapinar, A note on common fixed point theorems in partial metric spaces, Miskolc Math. Notes, 12 (2011), $185-191$.

[22] E. Karapınar, Generalizations of Caristi Kirk's theorem on partial metric spaces, Fixed Point Theory Appl., 2011 (2011), 7 pages.

[23] E. Karapınar, Ćirić types nonunique fixed point theorems on partial metric spaces, J. Nonlinear Sci. Appl., 5 (2012), 74-83. 1.6

[24] E. Karapınar, V. Berinde, Quadruple fixed point theorems for nonlinear contractions in partially ordered metric spaces, Banach J. Math. Anal., 6 (2012), 74-89.

[25] E. Karapınar, K. P. Chi, T. D. Thanh, A generalization of Ćirić quasicontractions, Abstr. Appl. Anal., 2012 (2012), 9 pages.

[26] E. Karapınar, İ. M. Erhan, Fixed point theorems for operators on partial metric spaces, Appl. Math. Lett., 24 (2011), 1894-1899.

[27] E. Karapinar, İ. M. Erhan, Cyclic contractions and fixed point theorems, Filomat, 26 (2012), 777-782.

[28] E. Karapınar, İ. M. Erhan, A. Öztürk, Fixed point theorems on quasi-partial metric spaces, Math. Comput. Modelling, 57 (2013), 2442-2448.

[29] E. Karapınar, I. M. Erhan, A. Y. Ulus, Fixed point theorem for cyclic maps on partial metric spaces, Appl. Math. Inf. Sci., 6 (2012), 239-244.

[30] E. Karapınar, V. Rakocević, On cyclic generalized weakly C-contractions on partial metric spaces, Abstr. Appl. Anal., 2013 (2013), 7 pages.

[31] E. Karapinar, S. Romaguera, Nonunique fixed point theorems in partial metric spaces, Filomat, 27 (2013), $1305-1314$.

[32] E. Karapınar, K. Sadarangani, Fixed point theory for cyclic $(\phi-\psi)$-contractions, Fixed Point Theory Appl., 2011 (2011), 8 pages.

[33] E. Karapinar, W. Shatanawi, K. Tas, Fixed point theorem on partial metric spaces involving rational expressions, Miskolc Math. Notes, 14 (2013), 135-142.

[34] E. Karapınar, N. Shobkolaei, S. Sedghi, S. M. Vaezpour, A common fixed point theorem for cyclic operators on partial metric spaces, Filomat, 26 (2012), 407-414. 1.13

[35] E. Karapinar, I. S. Yuce, Fixed point theory for cyclic generalized weak $\phi$-contraction on partial metric spaces, Abstr. Appl. Anal., 2012 (2012), 12 pages.

[36] E. Karapınar, U. Yüksel, Some common fixed point theorems in partial metric spaces, J. Appl. Math., 2011 (2011), 16 pages. 1

[37] M. S. Khan, M. Swaleh, S. Sessa, Fixed point theorems by altering distances between the points, Bull. Austral. Math. Soc., 30 (1984), 1-9. 1.7

[38] S. G. Matthews, Partial metric topology, Papers on general topology and applications, Flushing, NY, (1992), Ann. New York Acad. Sci., New York Acad. Sci., New York, 728 (1994), 183-197. 1, 1.1, 1.2, 1.3, 3, 3.3

[39] Z. Mustafa, M. Jaradat, A. Ansari, F. Gu, H.-H. Zheng, S. Radenović, Common fixed point results for four self-mappings in partial metric space using C-class functions on ( $\psi, \phi)$-contractive condition, (Submitted). 1.10, 1.11, 1.12

[40] H. K. Nashine, Z. Kadelburg, S. Radenović, Common fixed point theorems for weakly isotone increasing mappings in ordered partial metric spaces, Math. Comput. Modelling, 57 (2013), 2355-2365. 1.9

[41] H. K. Nashine, E. Karapınar, Fixed point results in orbitally complete partial metric spaces, Bull. Malays. Math. Sci. Soc., 36 (2013), 1185-1193. 1

[42] S. Oltra, O. Valero, Banach's fixed point theorem for partial metric spaces, Rend. Istit. Mat. Univ. Trieste, 36 (2004), 17-26. 1.2, 1.3, 1.5

[43] A. Roldán, J. Martínez-Moreno, C. Roldán, E. Karapınar, Multidimensional fixed-point theorems in partially ordered complete partial metric spaces under $(\psi, \phi)$-contractivity conditions, Abstr. Appl. Anal., 2013 (2013), 12 pages.

[44] S. Romaguera, A Kirk type characterization of completeness for partial metric spaces, Fixed Point Theory Appl., 2010 (2010), 6 pages. 1, 1.4

[45] W. Shatanawi, M. Postolache, Coincidence and fixed point results for generalized weak contractions in the sense of Berinde on partial metric spaces, Fixed Point Theory Appl., 2013 (2013), 17 pages. 1 